\title{
Personas mayores en aislamiento social en la ciudad de Madrid: experiencia de una intervención a través de la estrategia psicológica de búsqueda activa
}

\section{Older adults in social isolation in the city of Madrid: experience of an intervention using the psychological strategy of outreach}

\section{ANA BELÉN SANTOS-OLMO ${ }^{1}$ \\ BERTA AUSÍN ${ }^{1}$ \\ MANUEL MUÑOZ ${ }^{2}$ \\ PILAR SERRANO ${ }^{3}$}

\section{RESUMEN}

El objetivo del Servicio de Apoyo Psicológico a Ancianos Frágiles con Aislamiento Social en Madrid es lograr un acercamiento a las personas mayores con claro riesgo de aislamiento social, que permita conducir esas situaciones a la normalización, asignando los recursos sociales oportunos en cada caso e incluso facilitando el internamiento involuntario y considerando la incapacitación legal en último extremo.

Se describe en el artículo el Servicio, la metodología que emplea y los resultados obtenidos en los primeros 19 meses de existencia del mismo, en cuanto a personas atendidas. Por último, se describe la población en cuanto a salud y funcionamiento psicosocial, necesidades socio-sanitarias, funcionamiento global y discapacidad.

La presente investigación aplicada ha puesto de manifiesto la necesidad de contar con profesionales formados en estrategias psicológicas de búsqueda activa, enganche $y$ tratamiento que permita abordar con ciertas garantías los problemas de este grupo de población. Los datos indican que las intervenciones con este tipo de población necesitan

\footnotetext{
${ }^{1}$ Colegio Oficial de Psicólogos de Madrid.

${ }^{2}$ Facultad de Psicologia. Universidad Complutense de Madrid. mmunoz@psi.ucm.es

${ }^{3}$ Dirección General de Mayores de Madrid.
} 
de una duración prolongada, con intervenciones a medio y largo plazo (entre 3 y 15 meses). Conviene diferenciar entre tres grupos de intervención (Normalización, Contención y Custodia) en los que los tiempos de intervención son diferentes.

\title{
PALABRAS CLAVE
}

Aislamiento social, Personas mayores, Búsqueda activa, HoNOS65+, CANE.

\begin{abstract}
The aim of the Service of Psychological Support to Fragile Elders with Social Isolation in Madrid is to achieve an approximation to the older persons with clear risk of social isolation, which allows to lead these situations to the normalization, assigning the social opportune resources in every case and even facilitating the involuntary internment and considering the legal incapacitation in last end.

This article describes the Service, used methodology and the obtained results in the first 19 months of existence of the Service. Finally, the population is described in terms of health and psychosocial functioning, social and sanitary needs, global functioning and disability.

The present applied research has revealed the need of having professionals trained in psychological strategies of outreach, engage and treatment allowing to approach with certain guarantees the problems of this population group. The information indicates that the interventions with this type of population need a long duration, with interventions to medium and long term (between 3 and 15 months). It is convenient to make distinction between three groups of intervention (Normalization, Containment and Custody) in which the times of intervention are different.
\end{abstract}

\section{KEY WORDS}

Social isolation, Older adults, Outreach, HoNOS65+, CANE. 


\section{CONTEXTO DE LA APLICACIÓN}

En primer lugar, antes de avanzar en el estudio de los determinantes y los efectos de las situaciones de aislamiento y soledad, conviene llevar a cabo un comentario terminológico que ayude a entender mejor las complejas relaciones y a clarificar los posibles malentendidos semánticos unidos a los términos de exclusión social, aislamiento y soledad.

En primer lugar, exclusión social es un término claramente relacionado con los procesos estructurales, macroeconómicos y sociológicos que se comenzó a emplear por Jacques Delors (Presidente de la Comisión Europea 1985-1995) unido al concepto de exclusión del mercado laboral en el marco de la Unión Europea. Desde entonces su definición ha ido incorporando dominios de exclusión y en la actualidad se puede entender por exclusión social: la situación en que se encuentran las personas que no pueden participar plenamente en la vida económica, social y civil y cuyos ingresos o recursos (personales, familiares, sociales y culturales) son tan inadecuados que no les permite disfrutar de un nivel y una calidad de vida considerado aceptable por la sociedad en la que viven (Comisión Europea en el Informe conjunto sobre la Integración Social, Bruselas, 12/12/2001).

Un segundo grupo de términos hace referencia al concepto de soledad, término que proviene de la tradición psicológica y, por tanto, aglutina su significado en torno a los factores psicológicos. Soledad se ha definido como: el estado aversivo experienciado cuando existe una discrepancia entre las relaciones interpersonales deseadas y las percibidas en cada momento (Peplau y Perlman, 1982).

Esta conclusión conduce directamente a la necesidad de considerar el tercer término en cuestión: aislamiento. Térmi- no más próximo a los niveles microsociales, familiares, de grupo, amigos y, en definitiva, factores relacionales y psicosociales. El aislamiento ha sido definido de forma muy variada en la literatura científica confundiéndose frecuentemente con soledad y exclusión. En general, se habla de aislamiento social como la ausencia de relaciones sociales satisfactorias y de un nivel bajo de participación en la vida comunitaria. El aislamiento social implica experimentar una serie de factores como bajo nivel de participación social y niveles de actividad social que la persona percibe como inadecuada. Hall y Havens (1999) definieron el aislamiento social como la medida objetiva de tener minimas interacciones con otros; y aislamiento emocional (o soledad), como el sentimiento subjetivo de insatisfacción con el bajo número de contactos sociales mantenidos. El paralelismo entre soledad objetiva-soledad socialaislamiento social y soledad subjetivasoledad emocional-aislamiento emocional resulta obvio y para muchos autores configura el significado de ambos términos. En esta misma linea, muy recientemente Cattan, White, Bond et al. (2005) definen la soledad o aislamiento emocional como el sentimiento subjetivo de falta o pérdida de compañia, mientras que consideran que el aislamiento social es la ausencia objetiva de contactos y de interacciones entre la persona mayor y la red social.

En España hay cerca de 7 millones de personas mayores de 65 años, es decir, más de un $17 \%$ de la población total (Censo de Población, 2001). De estos, 1.358.937 $(19,53)$ viven solos. En cuanto a Madrid, las cifras indican que son 132.683 las personas mayores de 65 años que viven solas (Padrón de Habitantes a fecha de 1 de enero de 2007). A pesar de su vida en soledad, la gran mayoría de ellos se encuentra perfectamente integrada en la sociedad, goza de 
una buena calidad de vida y tiene los apoyos que precisa.

No obstante, en algunos casos se produce una suma de factores asociados a la soledad, como son la fragilidad, la dependencia funcional, la situación socio-familiar deficitaria, la presencia de problemas de salud, posibles alteraciones del comportamiento y el rechazo sistemático a las ayudas sociales, que puede llegar a definir un grupo de alto riesgo social y sanitario, haciendo necesario disponer de un sistema de detección, valoración e intervención específico al efecto. En este sentido, la Dirección General de Mayores del Ayuntamiento de Madrid, está llevando a cabo desde el año 2004 el Programa de Detección de Ancianos Frágiles con Alto Riesgo de Aislamiento Social, con el objetivo general de evitar las situaciones de aislamiento social de las personas mayores de 65 años del municipio de Madrid, así como las emergencias y los fallecimientos en riesgo de aislamiento social o en abandono.

En este contexto y dentro del Convenio de Colaboración entre el Área de Gobierno de Familia y Servicios Sociales y la Vocalía de Psicología de la Intervención Social del Colegio Oficial de Psicólogos de Madrid, surge el Servicio de Apoyo Psicológico a Ancianos Frágiles con Aislamiento Social en Madrid (SAP). Dicho Servicio está integrado por 2 psicólogas a tiempo completo con el apoyo de las trabajadoras sociales de los centros de Servicios Sociales Municipales. La coordinación del mismo se realiza desde la Universidad Complutense de Madrid, mientras que la responsabilidad del Servicio recae en la Dirección General de Mayores del Ayuntamiento de Madrid.

\section{OBJETIVOS DE INTERVENCIÓN}

El objetivo general del SAP es lograr un acercamiento a las personas mayores frágiles con claro riesgo de aislamiento 0 exclusión social que permita conducir esas situaciones a la normalización, asignando los recursos sociales oportunos en cada caso e incluso facilitando el internamiento involuntario y considerando la incapacitación legal en último extremo.

Los objetivos específicos son los siguientes:

1. Realizar una labor de enganche (búsqueda activa), de las personas en aislamiento social, con el fin de que enganchen 0 se re-enganchen con la red socio-sanitaria.

2. Realizar una evaluación lo más exhaustiva posible, según los casos, que incluya datos de valoración de la salud, funcionamiento psicosocial, nivel de discapacidad y necesidades no cubiertas.

3. Realizar una labor de intervención, siempre en coordinación con los Servicios Sociales de los distritos, con el objetivo de la normalización de la situación de la persona 0 , en último extremo, el apoyo al proceso de ingreso no voluntario e incapacitación legal.

4. Fortalecer la red social de la persona mayor en aislamiento, para que apoye el proceso de contacto/ enganche, evaluación e intervención.

5. Desestigmatizar a la persona en aislamiento, tanto en su vecindario como en su contexto familiar.

Las lineas directrices del SAP a Ancianos Frágiles con Aislamiento Social en Madrid se refieren a aspectos de la calidad, la búsqueda activa, el seguimiento 
de caso, la atención individual y la continuidad en la atención.

\section{METODOLOGÍA}

\section{Criterios de inclusión de la población objeto de atención:}

- Ser mayor de 65 años

- Vivir solo/a o con características de aislamiento especiales.

- Necesitar ayudas debido a:

- Problemas de salud física y funcionamiento que generan dependencia (hipoacusia, visión, movilidad y salud en general).

- Problemas de salud mental (depresión, deterioro cognitivo, trastorno mental crónico de larga duración).

- Disponer de una escasa red de apoyo social. (v.g. no tener a nadie a quien acudir para pedir ayuda).

- Rechazar todas las ayudas que se le ofrecen para cubrir sus necesidades desde los Servicios Sociales de distrito.

- Esta situación puede suponer un riesgo para sí mismo y/o para los demás.

- Desde los Servicios Sociales se han agotado todas las vías de intervención.

\section{Diseño de la intervención}

A continuación, se describe el procedimiento de intervención desde que se produce la derivación al SAP, hasta que se finaliza el seguimiento.
En cuanto a la derivación: son los centros de servicios sociales de los distintos distritos los que derivan los casos al SAP, utilizando una ficha de derivación especifica para este servicio.

$\mathrm{El}$ procedimiento de intervención tiene lugar siguiendo la estrategia psicológica de búsqueda activa y parte de cuatro principios básicos para establecer relaciones adecuadas: realismo; perspectiva a largo plazo; empatía y actitud positiva hacia la persona; flexibilidad e intervención basada en la persona.

La intervención se compone de los siguientes pasos:

- Primera fase: Actualización del caso: se trata de asegurarse de que la situación de la persona mayor es de aislamiento social en el momento en que el SAP comience su intervención. En esta fase, es básico que la valoración se haga con el trabajador social de referencia.

- Segunda fase:

- Protocolo de contacto y enganche: Los contactos con cada persona usuaria y su entorno adoptarán algunas de estas modalidades: Visitas tanto a la persona mayor en su domicilio, en la calle o en lugares de su comunidad frecuentados por él, como a personas de su red informal (familiares, vecinos, amigos, porteros, etc) y formal (médico, enfermeria, profesionales de los centros de mayores, etc); llamadas telefónicas a la persona usuaria o a personas de su red informal y formal; etc.

- Protocolo de evaluación: se evalúa psicológicamente el estado de la persona en cuanto a las siguientes variables: 
- Salud y Funcionamiento psicosocial, a través del empleo de las HoNOS65+ Escalas de Evolución para Personas Mayores de 65 años (Burns, Beevor, Lelliott et al., 1999. Adaptación de Ausin, Muñoz y Quiroga, 2007).

- Necesidades socio-sanitarias, empleando el CANE Cuestionario de Evaluación de Necesidades en Ancianos de Camberwell (Reynolds, Thornicroft, Abas et al., 2000).

- Funcionamiento global, utilizando el EEFG Escala de Evaluación del Funcionamiento Global (Asociación Americana de Psiquiatría, 2002).

- Discapacidad, a través del WHO-DAS Escala de Discapacidad de la OMS (Organización Mundial de la Salud, 2000).

- Otras variables en función de las características específicas de la persona: depresión, sintomatología psiquiátrica, deterioro cognitivo, etc. a través del BPRS Escala Breve de Valoración de Síntomas Psiquiátricos (Overall y Gorham, 1962); Mini examen del estado mental (Folstein, Folstein y McHugh, 1975).

- Protocolo de intervención: Tras esta evaluación psicológica, el SAP diseña el plan individual de actuación (PIA) en coordinación con el trabajador social de zona de los Servicios Sociales del distrito correspondiente. El diseño del plan individual de atención se basa en las necesidades detectadas en la persona, así como en la posibilidad de recibir diferentes ayudas y recursos. En general, el proceso de evaluación lleva a distinguir entre tres grupos de necesidades que pueden configurarse como grupos de intervención diferente: grupo de normalización (incluye aquellos casos que son susceptibles de normalización); grupo de contención o mantenimiento (incluye aquellos casos en los que ni la normalización ni la custodia son las vías de intervención adecuadas); grupo de custodia (incluye aquellos casos cuyo nivel de deterioro es irrecuperable). En este último grupo de intervención, el SAP elabora un informe de valoración psicológica que se adjuntará junto con el resto de documentación necesaria, a través de los Servicios Sociales, a Fiscalía o a los Juzgados.

- Tercera fase: Seguimiento: se realiza un seguimiento de cada caso para evaluar que las necesidades de la persona continúan cubiertas dentro de los 3 a 6 meses posteriores a la intervención.

\section{RESULTADOS DEL SERVICIO DE APOYO PSICOLÓGICO A ANCIANOS FRÁGILES CON AISLAMIENTO SOCIAL EN MADRID (SAP)}

\section{Personas atendidas:}

Al servicio han llegado a lo largo de 19 meses un total de 143 personas, de las cuales se han descartado 43 por no cumplir los criterios de inclusión. De este modo, la población final está constituida por 100 personas mayores en aislamiento social en Madrid.

Distribución por perfiles

Teniendo en cuenta los tres perfiles de intervención, y tomando como muestra 
los casos de personas mayores en aislamiento social cerrados por el Servicio de Apoyo Psicológico $(\mathrm{N}=36)$, cabe destacar lo siguiente (tabla 1):

- Perfil 1 o de Normalización: el $16,7 \%$ de los casos en los que se ha intervenido pertenece a este grupo. El tiempo de intervención medio con este grupo es de 3 meses, con una media de contactos de 11,83 .

- Perfil 2 o de Contención: el 25\% de los casos en los que se ha intervenido pertenece a este grupo. El tiempo de intervención medio con este grupo es de 6,90 meses, con una media de contactos de 36,22.

- Perfil 3 o de Custodia: el 58,3\% de los casos en los que se ha intervenido pertenece a este grupo. El tiempo de intervención medio con este grupo es de 3,76 meses, con una media de contactos de 13,24. A lo largo de estos 19 meses de trabajo, el SAP ha redactado 21 informes de valoración de la salud, funciona- miento psicológico y social para Fiscalia de Mayores.

Teniendo en cuenta los tres perfiles de intervención, y tomando como muestra los casos de personas mayores en aislamiento social activos a 31 de diciembre de 2007 en el Servicio de Apoyo Psicológico $(\mathrm{N}=27)$, cabe destacar lo siguiente (tabla 2):

- Perfil 1 o de Normalización: el $18,2 \%$ de los casos en los que se está interviniendo pertenece a este grupo. El tiempo de intervención medio con este grupo es de 14,50 meses, con una media de contactos de 52,25 .

- Perfil 2 o de Contención: el 27,3\% de los casos en los que se está interviniendo pertenece a este grupo. El tiempo de intervención medio con este grupo es de 9,33 meses, con una media de contactos de 22,67.

- Perfil 3 o de Custodia: el 54,5\% de los casos en los que se está interviniendo pertenece a este grupo. El

Tabla 1. Casos en aislamiento cerrados por el Servicio De Apoyo Psicológico: Grupos de intervención (porcentajes, tiempo de intervención y número de contactos)

\begin{tabular}{|c|c|c|c|}
\hline $\begin{array}{l}\text { Grupo de } \\
\text { Intervención } \\
(\mathrm{n}=36)\end{array}$ & Casos & $\begin{array}{c}\text { Tiempo de } \\
\text { intervención } \\
\text { (en meses) }\end{array}$ & $\begin{array}{l}\text { Número de } \\
\text { contactos }\end{array}$ \\
\hline Normalización & $\begin{array}{c}6 \\
16,7 \%\end{array}$ & $\begin{array}{c}\text { Media: } 3 \\
\text { Moda: } 3 \\
\text { Desviación típica: } \\
1,27 \\
\text { Rano: } 1-5\end{array}$ & $\begin{array}{c}\text { Media: } 11,83 \\
\text { Moda: } 11 \\
\text { Desviación típica: } \\
6,55 \\
\text { Ranoo: } 4-24\end{array}$ \\
\hline Contención & $25 \%$ & $\begin{array}{c}\text { Media: } 6,90 \\
\text { Moda: } 7 \\
\text { Desviación típica: } \\
4,08 \\
\text { Rango: } 1-14\end{array}$ & $\begin{array}{c}\text { Media: } 36,22 \\
\text { Moda: } 8 \\
\text { Desviación típica: } \\
28,9 \\
\text { Rango: } 8-74\end{array}$ \\
\hline Custodia & $\begin{array}{c}21 \\
58,3 \%\end{array}$ & $\begin{array}{c}\text { Media: } 3,76 \\
\text { Moda: } 1 \\
\text { Desviación típica: } \\
\text { 3,08 } \\
\text { Rango: } 1-10\end{array}$ & $\begin{array}{c}\text { Media: } 13,24 \\
\text { Moda: } 1 \\
\text { Desviación tipica: } \\
12,14 \\
\text { Rango: } 1-40\end{array}$ \\
\hline
\end{tabular}


tiempo de intervención medio con este grupo es de 4,17 meses, con una media de contactos de 15,50. A lo largo de estos 19 meses de trabajo, el SAP ha redactado 21 informes de valoración de la salud, funcionamiento psicológico y social para Fiscalía de Mayores. cia). Han sido 36 los expedientes cerrados por este Servicio tras la fase de intervención, es decir, 68 personas han dejado de estar en situación de aislamiento social. En este sentido, los motivos de cierre han sido diversos (tablas 3 y 4).

Descripción de las personas atendidas:

Tabla 2. Casos en aislamiento activos a 31 de diciembre de 2007: Grupos de intervención (porcentajes, tiempo de intervención y número de contactos)

\begin{tabular}{|c|c|c|c|}
\hline $\begin{array}{c}\text { Grupo de } \\
\text { Intervención }(\mathrm{n}=27) \\
\left({ }^{*}\right)\end{array}$ & Casos & $\begin{array}{c}\text { Tiempo de } \\
\text { intervención } \\
\text { (en meses) }\end{array}$ & Número de contactos \\
\hline Normalización & $\begin{array}{c}4 \\
18,2 \%\end{array}$ & $\begin{array}{c}\text { Media: } 14,50 \\
\text { Moda: } 17 \\
\text { Desviación tipica: } 5 \\
\text { Rango: } 7-17\end{array}$ & $\begin{array}{c}\text { Media: } 52,25 \\
\text { Moda: } 54 \\
\text { Desviación típica: } \\
31,5 \\
\text { Rano. }\end{array}$ \\
\hline Contención & $\begin{array}{c}6 \\
27,3 \%\end{array}$ & $\begin{array}{c}\text { Media: } 9,33 \\
\text { Moda: } 9 \\
\text { Desviación típica: 4,6 } \\
\text { Rango: } 1-14\end{array}$ & $\begin{array}{c}\text { Rango: } 12-89 \\
\text { Media: } 22,67 \\
\text { Moda: } 6 \\
\text { Desviación típica: } \\
12,1 \\
\text { Rango: } 6-38\end{array}$ \\
\hline Custodia & $\begin{array}{c}12 \\
54,5 \%\end{array}$ & $\begin{array}{c}\text { Media: } 4,17 \\
\text { Moda: } 2 \\
\text { Desviación típica: } \\
2,66 \\
\text { Rango: } 1-8\end{array}$ & $\begin{array}{c}\text { Media: } 15,50 \\
\text { Moda: } 18 \\
\text { Desviación típica: } \\
6,17 \\
\text { Rango: } 4-23\end{array}$ \\
\hline
\end{tabular}

* 5 personas no tienen asignación de grupo (se está evaluando el grupo de intervención)

Casos cerrados y motivos de cierre:

Del total de 100 personas mayores atendidas, 32 expedientes se han cerrado en la primera fase previa al inicio de la intervención, la fase de actualización (la razón principal es que la persona mayor ya estaba ingresada en residen-
Datos socio-demográficos: Sexo, edad, estado civil, hijos, convivencia:

En la tabla 5 se recoge la distribución de las personas mayores en aislamiento social por sexos, en cuanto a las distintas variables sociodemográficas estudiadas.

\section{Tabla 3. Motivos de cierre en la fase de actualización}

\begin{tabular}{|lc|}
\hline Casos cerrados en la 1 a Fase: ACTUALIZACION & 5 \\
\hline Fallecimiento & 13 \\
Ingreso residencia & 1 \\
Traslado a otra Comunidad Autónoma & 4 \\
Implicación familiares & 1 \\
Cuidador / auxiliar privado & 2 \\
Acepta recursos & 6 \\
Ingreso no voluntario a corto plazo (sin informe del SAP) & 32 \\
Total & \\
\hline
\end{tabular}


Tabla 4. Motivos de cierre en la fase de intervención

\begin{tabular}{|lc|}
\hline Casos cerrados en 2 ${ }^{-}$Fase: INTERVENCION. & 5 \\
\hline Acepta recursos & 3 \\
Traslado a otra Comunidad Autónoma & 4 \\
Ingreso no voluntario a corto plazo (sin informe del SAP) & 3 \\
Fortalecimiento de la red de apoyo por intervención del SAP & 19 \\
Apoyo a ingreso no voluntario en residencia (informe) & 2 \\
Normalización previa + Apoyo a ingreso no voluntario en residencia (informe) & 6 \\
Total & \\
\hline
\end{tabular}

De las 100 personas en aislamiento, 69 son mujeres $(69 \%)$ y 31 hombres (31\%).

En cuanto a la variable edad, la persona más joven tiene 67 años, y la mayor 97 , siendo la edad media de 79,73 años, moda de 80 y desviación típica de 7,30 .

Se observa en la tabla 6, que la edad media de los hombres y las mujeres no es muy diferente, siendo las mujeres de la población estudiada algo mayores que los hombres (edad media de las mujeres de 80,28; y de los hombres 78,39).
En cuanto al estado civil, el $13,4 \%$ de las personas están casadas, $47,4 \%$ solteras, $35,1 \%$ son viudas. El menor porcentaje lo ocupan las personas separadas / divorciadas, con un $4,1 \%$.

En la tabla 5 se recoge la distribución por sexos en cuanto a la variable estado civil, y hay mayor porcentaje de hombres separados o divorciados. Se encuentran también diferencias en cuanto al porcentaje de mujeres viudas, bastante más alto que el de hombres viudos.

El 72,7\% de la muestra de personas en aislamiento social en Madrid viven solas

Tabla 5. Descripción sociodemográfica del total de la población y su distribución por sexos

\begin{tabular}{|c|c|c|c|}
\hline Variable & Total & Hombre & Mujer \\
\hline $\begin{array}{l}\text { Sexo } \\
(\mathrm{n}=100)\end{array}$ & $100 \%$ & $31 \%$ & $69 \%$ \\
\hline Edad media & 79,73 & 78,39 & 80,28 \\
\hline \multicolumn{4}{|l|}{$\begin{array}{l}\text { Estado civil } \\
(\mathrm{n}=97)\end{array}$} \\
\hline Casado/a & $13,4 \%$ & $20 \%$ & $10,4 \%$ \\
\hline Soltero/a & $47,4 \%$ & $50 \%$ & $46,3 \%$ \\
\hline Viudo/a & $35,1 \%$ & $23,3 \%$ & $40,3 \%$ \\
\hline Separado/a o divorciado/a & $4,1 \%$ & $6,7 \%$ & $3 \%$ \\
\hline \multicolumn{4}{|l|}{$\begin{array}{l}\text { Convivencia } \\
(\mathrm{n}=99)\end{array}$} \\
\hline Esposo/a & $10,1 \%$ & $16,7 \%$ & $7,2 \%$ \\
\hline Solo/a & $72,7 \%$ & $70 \%$ & $73,9 \%$ \\
\hline Hijo/a & $7,1 \%$ & $3,3 \%$ & $8,7 \%$ \\
\hline Hermano/a & $8,1 \%$ & $6,7 \%$ & $8,7 \%$ \\
\hline Amigo/a & $2 \%$ & $3,3 \%$ & $1,4 \%$ \\
\hline \multicolumn{4}{|l|}{$\begin{array}{l}\text { Hijos } \\
(\mathrm{n}=97)\end{array}$} \\
\hline Con hijos & $27.8 \%$ & $30 \%$ & $26,9 \%$ \\
\hline Con hijos fallecidos & $2,1 \%$ & 0 & $3 \%$ \\
\hline Sin hijos & $70,1 \%$ & $70 \%$ & $70,1 \%$ \\
\hline
\end{tabular}


en el momento de derivación al Servicio de Apoyo Psicológico. Algunas personas de la muestra, a pesar de estar aisladas, conviven con otras personas (el 10,1\% con el esposo/a, el 7,1\% con un hijo/a, el $8,1 \%$ con un hermano/a y el $2 \%$ con un amigo/a). En la tabla 5 se observa que las mujeres conviven en un porcentaje mayor con un hijo/a, o con un hermano/a. Mientras que los hombres conviven en un porcentaje mayor con un amigo/a.

No tener hijos parece ser un factor de vulnerabilidad de la situación de aislamiento social, dado que el $70,1 \%$ de la muestra no tiene hijos.

Descripción de la población en cuanto a Salud y funcionamiento psicosocial, Necesidades socio-sanitarias, Funcionamiento global y Discapacidad:

De las 100 personas en aislamiento social en Madrid incluidas en el Servicio de Apoyo Psicológico, se cuenta con valoraciones de 51 personas. Esto se debe a que el Servicio de Apoyo Psicológico ha trabajado con casos que se han cerrado antes de la fase de evaluación, y con otros, la intervención acaba de comenzar, por lo que no se cuenta con información suficiente para cumplimentar estas escalas de evaluación.
- Valoración salud y funcionamiento psicosocial.

Se emplearon las Escalas de Evolución para Personas Mayores de 65 años HoNOS65+ (Burns et al., 1999; Ausin et al., 2007).

En la tabla 6 se recoge la proporción de personas que presentan cada uno de los 12 problemas, a un nivel moderado 0 grave. Se observa cómo más de la mitad de las personas presenta los siguientes problemas: discapacidad física, problemas con las relaciones sociales, problemas con las condiciones de vida y problemas de ocio.

- Valoración de las necesidades sociosanitarias.

Se empleó el Cuestionario de Evaluación de Necesidades en Ancianos de Camberwell CANE (Reynolds et al., 2000).

En la tabla 7 se recoge un resumen de las puntuaciones encontradas. Se observa que la población de personas mayores en aislamiento social en Madrid presenta un número muy elevado de necesidades sin cubrir.

\section{Tabla 6. Escalas HoNOS65+ en la población. Porcentaje que puntúa con problema de moderado a grave}

\begin{tabular}{|lc|}
\hline \multicolumn{1}{|c|}{ Escalas HoNOS65+ } & Total \\
\hline Escala 1. Conducta Agitada (n = 51) & $41,2 \%$ \\
Escala 2. Autolesiones (n = 51) & $2 \%$ \\
Escala 3. Alcohol (n = 46) & $8,7 \%$ \\
Escala 4. Cognición (n = 46) & $32,6 \%$ \\
Escala 5. Fisico / discapacidad (n = 49) & $57,1 \%$ \\
Escala 6. Alucinaciones /delirios (n = 48) & $29,2 \%$ \\
Escala 7. Depresión (n = 47) & $8,3 \%$ \\
Escala 8. Otros problemas (n = 45) & $45,5 \%$ \\
Escala 9. Relaciones sociales (n = 51) & $91,7 \%$ \\
Escala 10. Actividades Vida Diaria (n $=50)$ & $91,7 \%$ \\
Escala 11. Condiciones vida (n = 51) & $75 \%$ \\
Escala 12. Ocio/entorno (n = 51) & $83,3 \%$ \\
\hline
\end{tabular}


Tabla 7. Puntuaciones escala CANE en la población. Porcentaje de necesidades no cubiertas

\begin{tabular}{|c|c|}
\hline CANE & Total \\
\hline Área 1. Alojamiento $(n=51)$ & $66,7 \%$ \\
\hline Area 2. Cuidado del hogar $(n=51)$ & $74,5 \%$ \\
\hline Ârea 3. Alimentaciôn $(n=46)$ & $34,8 \%$ \\
\hline Area 4. Cuidado personal $(n=50)$ & $76 \%$ \\
\hline Area 5. Cuidado de otra persona $(n=51)$ & $13,7 \%$ \\
\hline Arrea 6. Actividades diarias $(n=51)$ & $86,3 \%$ \\
\hline Ârea 7. Memoria $(n=45)$ & $44,4 \%$ \\
\hline Ârea 8. Vista / oído $(n=47)$ & $38,3 \%$ \\
\hline Ârea 9. Movilidad $(n=48)$ & $20,8 \%$ \\
\hline Ârea 10. Continencia $(n=33)$ & $30,3 \%$ \\
\hline Ârea 11. Salud fisica $(n=44)$ & $47,7 \%$ \\
\hline Ârea 12. Medicaciôn $(\mathrm{n}=39)$ & $51,3 \%$ \\
\hline Ârea 13. Sintomas psicóticos $(n=48)$ & $29,2 \%$ \\
\hline Ârea 14. Angustia ( $\mathrm{n}=47)$ & $21,3 \%$ \\
\hline Ârea 15. Información sobre su enfermedad y tratamiento $(n=47)$ & $27,7 \%$ \\
\hline Área 16. Riesgo para sí mismo (auto agresión) $(n=49)$ & $4,1 \%$ \\
\hline Ârea 17. Riesgo para si mismo (accidentes) $(n=51)$ & $80,4 \%$ \\
\hline Ârea 18. Seguridad (abuso / abandono) $(n=48)$ & $39,6 \%$ \\
\hline Ârea 19. Conducta / riesgo para los demás $(n=51)$ & $72,5 \%$ \\
\hline Area 20. Alcohol $(n=46)$ & $10,9 \%$ \\
\hline Ârea 21. Compañía $(n=51)$ & $90,2 \%$ \\
\hline Area 22. Relaciones de pareja $(n=51)$ & $3,9 \%$ \\
\hline Arrea 23. Manejo del dinero $(n=48)$ & $25 \%$ \\
\hline Area 24. Prestaciones sociales $(n=42)$ & $11,9 \%$ \\
\hline
\end{tabular}

${ }^{*} p<0,1{ }^{* *} p<0,05{ }^{* * *} p<0,01$

- Valoración del funcionamiento global.

Esta puntuación indica un grado de fun-

Se empleó la Escala de Evaluación del Funcionamiento Global EEFG (Asociación Americana de Psiquiatría, 2002).

La muestra $(n=51)$ presenta puntuaciones que oscilan entre 15 y 85 puntos en esta escala. La media es de 44,31. cionamiento bajo a muy bajo (tabla 8).

- Valoración de la discapacidad.

Se empleó la Escala de Discapacidad de la OMS WHO-DAS (OMS, 2000).

Más del $80 \%$ de las personas estudia-

Tabla 8. Puntuación media en la escala EEFG de funcionamiento global en la población total

\begin{tabular}{|cc|}
\hline EEFG & Media \\
\hline EEFG $(\mathrm{n}=51)$ & 44,31 \\
\hline
\end{tabular}

${ }^{*} p<0,1 \quad{ }^{* *} p<0,05{ }^{* * *} p<0,01$

Tabla 9. Porcentaje de personas con discapacidad de obvia a máxima en la escala DAS en la población total

\begin{tabular}{|lc|}
\hline \multicolumn{1}{|c|}{ Escala DAS } & Total \\
\hline Escala A. Cuidado personal $(\mathrm{n}=50)$ & $84 \%$ \\
Escala B. Funcionamiento ocupacional $(\mathrm{n}=50)$ & $86 \%$ \\
Escala C. Funcionamiento familiar $(\mathrm{n}=38)$ & $92,1 \%$ \\
Escala D. Funcionamiento en el contexto social amplio $(\mathrm{n}=51)$ & $94,1 \%$ \\
\hline
\end{tabular}

${ }^{*} p<0,1{ }^{* *} p<0,05{ }^{* * *} p<0,01$ 
das muestran una discapacidad de obvia a máxima en las 4 subescalas de la DAS (tabla 9).

\section{CONCLUSIONES}

El presente trabajo ha puesto de manifiesto el valor añadido que supone la colaboración entre instancias municipales, responsables de la atención directa a las personas mayores, colegios profesionales y la Universidad, creando equipos de colaboración y trabajo conjunto que han permitido conjugar los aspectos más aplicados con las exigencias del trabajo de investigación. A continuación, se presentan las principales conclusiones de cada uno de los puntos esenciales.

De forma complementaria, el análisis cualitativo ha evidenciado la conveniencia de proceder a su atención desde una optica distinta a la seguida hasta el momento en los servicios existentes. Se ha puesto de manifiesto la necesidad de contar con profesionales formados en estrategias psicológicas de búsqueda activa, enganche y tratamiento que permita abordar con ciertas garantías los problemas de este grupo de población. En este sentido, un logro importante del trabajo ha consistido en demostrar la posibilidad real de diseñar y poner en funcionamiento un servicio de atención psicológica a las personas mayores en aislamiento social y su inserción en la red normalizada municipal de recursos para personas mayores.

Los datos indican que las intervenciones con este tipo de población necesitan de una duración prolongada, con intervenciones a medio y largo plazo (entre 3 y 15 meses). No obstante, la evaluación del proceso de este Servicio pone de manifiesto la conveniencia de diferenciar entre tres grupos de población en función del tipo de intervención que requieren (Normalización, Contención y Custodia), en los que los tiempos de intervención son diferentes. En este sentido, los casos susceptibles de normalización requieren, tanto un tiempo mayor de intervención como un mayor número de contactos antes de ser cerrados, mientras que el grupo de custodia requiere un menor tiempo de intervención y un número menor de contactos. 


\section{BIBLIOGRAFÍA}

Asociación Psiquiátrica Americana (2002). DSM-IV-TR. Barcelona: Masson

Ausín, B., Muñoz, M. y Quiroga, M.A. (2007). Adaptación Española De Las Escalas De Resultados Para Personas Mayores Honos65+ (Health Of The Nation Outcome Scales For Older Adults). Revista española de geriatría y gerontologia: Órgano oficial de la Sociedad Española de Geriatria y Gerontología, 42, 88-95.

Burns, A., Beevor, A., Lelliott, P., Wing, J., Blakey, A., Orrell, M., Mulinga, J. y Hadden, S. (1999). Health of the Nation Outcome Scales for Elderly People (HoNOS65+). Glossary for HoNOS65+ score sheet. British Journal of Psychiatry, 174, 435-438.

Cattan, M., White, M., Bond, J. y Learmouth, A. (2005). Preventing social isolation and loneliness among older people: a systematic review of health promotion interventions. Ageing and Society, 25, 41-67.

Censo de Población (2001). www.ine.es

Folstein, M.F., Folstein, S.E., McHugh, P.R. (1975). Mini-Mental State: A Practical Method for
Grading The Cognitive State of Patients for the Clinician. Journal of Psychiatric Research, 12, 189-198.

Hall, M. y Havens, B. (1999). Aging in Manitoba Study. Winnipeg: University of Manitoba.

Organización Mundial de la Salud (2002). Active aging a policy framework. Génova: OMS.

Overall, J.E. y Gorham, D.R. (1962). The Brief Psychiatric Rating Scale (BPRS). Psychol Rep, 10, 799-812.

Padrón de Habitantes a fecha de 1 de enero de 2007 (2007). www.munimadrid.es

Peplau, L.A. y Perlman, D. (1982). Perspectives on loneliness. En L.A. Peplau y D. Perlman (Eds.), Loneliness: A sourcebook of current theory, research and therapy (pág. 1-18). Nueva York: Wiley.

Reynolds, T., Thornicroft, G., Abas, M., Woods, B., Hoe, J., Leese, M. y Orrell, M. (2000). Camberwell Assessment of Needs for the Elderly (CANE). Development, validity and reliability. British Journal of Psychiatry, 176, 444-452. 